\title{
Mythe und Staat in Bantu-Afrika
}

\author{
Von HARALD VON SICARD
}

I.

Das Generalthema unseres Symposions ist „Die Mythe vom Staat“. Hält man sich ganz strikt an die etymologische und historische Bedeutung dieser beiden Worte, dann muss ich freilich sofort bekennen, dass wenigstens Bantu-Afrika nicht in diesen Zusammenhang gehört. Vergessen wir nicht, dass der Begriff „Staat“ sehr jungen Datums ist und erst aus der Zeit des Absolutismus stammt ${ }^{1}$. Auf lateinisch wird das Wort bekanntlich bezeichnenderweise mit res publica oder civitas wiedergegeben, und das ,Imperium romanum" hatte im Unterschied hierzu einen ganz anderen Klang — weil es einen anderen Inhalt hatte. Falls mich mein afrikanisches Empfinden nicht trügt, hatte es weniger mit dem imperator zu tun, als mit mehr oder weniger bestimmten geographischen Grenzen, innerhalb derer das $j u s$ romanum obwaltete. In Russland tauchte der Begriff ,gosudarstvo" meines Wissens auch erst im Zusammenhang mit dem Absolutismus auf. Gosudap' ist der Herr und Herrscher, und gewissermassen ist es eine Ironie der Geschichte, dass der 'Term gosudarstvo im Kommunismus weiterlebt, allerdings ungefähr mit dem Inhalt des Begriffes imperium.

Sieht man sich nun nach Verbindungslinien zwischen diesem europäischen Staat und eventuellen Entsprechungen in Bantu-Afrika um, dann fällt einem ganz ungesucht ein Ausspruch des Roi le Soleil ein: „L'état c'est moi!" und dazu die Formulierung der Mitteilung beim Tode des Herrschers: „Le Roi est mort. Vive le Roi!" Das macht zunächst so stark den Eindruck eines Mythos vom afrikanischen Staat, wie das nur irgend möglich ist. Denn „Staat" heisst in Bantu-Afrika, um es ganz allgemein zu nehmen, vu-she, d. h. Herrschaft, Königtum, gosudarstvo. She ist „Herrscher, Häuptling“‘2, und $v u$ - ist das abstrahierende Präfix.

1 F. Kluge, Etymologisches Wörterbuch der deutschen Sprache, Berlin I957, S. 735.

2 Vgl. Anthropos, 54, 1959, S. 300: I. 
Aber damit sind die Entsprechungen auch ungefähr erschöpft, und alles wird nur zum Zerrbild, falls wir, was leider immer noch häufig geschieht, den afrikanischen Mythos in europäische Formen zwängen wollen, mögen sie noch so moderne soziologische Eintagsfliegen sein.

Der Herrscher ist für den afrikanischen Staat konstitutiv, allerdings nicht als Individuum (gibt es überhaupt so etwas?), sondern als Glied einer Kette von Generationen. Eike Haberland spricht einmal treffend von der Auffassung vom sakralen Königtum als einer ,,von der Person seines Trägers unabhängigen Institution"1. Hier liegt noch ein letzter Anklang von Entsprechung zum europäischen Mythos vor, der zuletzt nur doch unbewusst in der Wiederholung seiner Herrschernamen weiterlebte, der vielen Louis, Karl usw., denn der Name ist nicht nur Name, sondern soll zugleich die Fortsetzung des Wesens zum Ausdruck bringen. Während jedoch „L'état c'est moil" der Ausdruck höchster Selbstherrlichkeit ist, ist diese Art des konstitutiven Elementes beim vushe undenkbar. Da verhilft uns ein anderer europäischer Ausdruck zum besseren Verständnis afrikanischer Verhältnisse: "Wir, Kaiser (oder König) so und so von Gottes Gnaden". Das klingt christlich oder biblisch, und ist $z$. $T$. noch bis zuletzt christlich gefärbt, hat jedoch nichts mehr mit Christus zu tun, sondern führt an jene verschwommene Grenze heran, hinter welcher das Reich des Mythos beginnt.

Nach diesen einschränkenden Bemerkungen können wir es wagen, Mythe und Staat in Bantu-Afrika mit einigen konkreten Zügen bis in ihre Anfänge zu beleuchten.

II.

I. Das Wahlkönigtum, wie es etwa bei den Vimbundu in Südangola ${ }^{2}$ und bei den Südlichen Rozwi in Rhodesien ${ }^{3}$ und auch sonst als gewöhnlichste Form des Königtums in Bantu-Afrika vorkam, aber z. B. auch den SüdKuschiten bekannt ist ${ }^{4}$, ist es oft in hohem Grade mit dem Mythos verquickt und von ihm unterbaut. Wo als Kandidaten nur nächste Verwandte

${ }^{1}$ E. Haberland, „Das heilige Königtum“, Völkerkunde, hrsg. von B. Freudenfeld, r960, S. 79 .

2 A. Hauenstein, Les Hanya (Studien zur Kulturkunde, r9), Wiesbaden I967, S. 237.

3 Z. B. F. W. T. Posselt, Fact and Fiction, Bulawayo I935, S. 446.

4 H.-G. Schinkel, Haltung, Zucht und Pflege des Viehs bei den Nomaden Ost- und Nordostafrikas (Veröffentlichungen des Museums für Völkerkunde zu Leipzig, 21), Berlin 1970, S. 44.

9-724135 H. Biezais 
des Herrschers infrage kommen, kann natürlich auch der Mythos stärker in Erscheinung treten. So erhoben z. B. die Rozwi in Rhodesien den Anspruch, das Land nicht deshalb zu regieren, weil sie es erobert hatten, sondern als Gesandte Gottes, Mwaris, und in seinem Namen'1. Das wiederum hängt mit dem gesunden, nicht nur afrikanischen Empfinden zusammen, dass es Dinge gibt, die nicht Besitz des Menschen werden können, die ausserhalb des wirtschaftlichen Gebietes, in dem alles in Geld umgewechselt werden kann, liegen, die nur als Gabe eines höheren Wesens, dem sie gehören, zur Nutzniessung vergeben und verwaltet werden. Für das Empfinden des Jägers gehören hierher etwa die Tiere des Waldes, besonders die Herdentiere, die Eigentum der Herrin oder des Herrn der Tiere sind, welche die Erlegung nur eines von ihnen immer wieder gestatten. Hierher gehört die Frau als Quelle des Lebens — von „Brautpreis“ und „Brautkauf“ kann nur der Abendländer mit seiner vom Gelde, von Wirtschaft und Handel bestimmten und dadurch verzerrten Kultur reden - und der Afrikaner ist von ihr weithin angesteckt worden, und so „kauft" er sich heute seine Farmen. Zur Kategorie der Dinge, die nicht gekauft werden können, gehört nun aber gerade auch das Land, denn das Land ist Gottes². Land kann kein Mensch besitzen, kein Mächtiger mit noch so grossen Kriegerhaufen sich $\mathrm{zu}$ eigen erobern, kein noch so Reicher kaufen. Land und Geld schliessen einander prinzipiell aus. Dazu kommt noch, dass den grössten Anspruch auf ein Gebiet nicht etwa der jeweilige Herrscher und seine Häuptlinge haben, sondern jener Mensch, der sich auf ihm als erster ansiedelte. Auf seinen Geist muss auch der grösste Häuptling Rücksicht nehmen.

„,Da haben wir es!“ denkt befriedigt ein Europäer: „Religion ist also doch in eine politische Angelegenheit abgeglitten und wurde zu politischen Zwecken benutzt!" Ganz so einfach verhält es sich jedoch nicht, denn die Rozwi unterwarfen sich selbst Mwari, und einen Herrschersohn, der das nicht tat und Mwaris Gebote missachtete, kennt die 'Tradition nur als verabscheuten Apostaten ${ }^{3}$.

${ }^{1}$ C. Bullock, The Mashona, Cape Town and Johannesburg 1927, S. 40.

2 Vgl. H. v. Sicard, „Reiche und Königtum der Rozwi vom Ende des 17 . bis zum Anfang des 19. Jahrhunderts", Festschrift fiir Ad. E. Fensen, 2, 1964, S. 655; M. L. Daneel, The Background and Rise of Southern Shona Independent Churches, S-Gravenhage 1971 , S. 88.

${ }^{3}$ Vgl. A. M. Sebina, „Makalaka“, African Studies, 6, r947, S. 83. 
Wo bleibt nun bei alledem ,L'état c'est moi“"? Und von einem Erstarken der Zentralgewalt eines Reichsgebildes' kann man in Bantu-Afrika nur in Ausnahmefällen sprechen, und es scheint dann immer auf hervorragenden Persönlichkeiten zu beruhen und daher nur von kurzer Dauer gewesen zu sein. Als einmalige gewaltige Gestalten haben sie den Mythos bereichert, also gewissermassen in der hier zur Diskussion stehenden entgegengesetzter Richtung gewirkt, nämlich der Unterbauung des Staates durch den Mythos gedient.

2. Deutlich bricht der Mythos vom Staat bei den Rozwi und einer ganzen Reihe von Bantu-Völkern in den Erzählungen vom Feuerbringer oder vom heiligen Staatsfeuer durch. Das Mythologem vom Feuerbringer kann natürlich von sehr verschiedenen Gesichtspunkten betrachtet werden. Wir haben es hier bewusst mit demjenigen vom Staatsfeuer zusammengestellt. H. Baumann hat darauf hingewiesen, dass in Afrika zugewanderte Jäger oft als Dynastiegründer vorkommen². Sie legen den Grund zum Herrschaftssystem einer fremdstämmigen Adelsschicht über autochthone Vasallen, wie das z. B. auch in Erythräa vorkommt3 ${ }^{3}$, was dann wieder mythologische Begründungen des Herrschersystems fördert. Man denke etwa an die Mythe vom ersten Loango-König, dem Sohn des grossen Jägers Nkungu, d. h. des ersten Menschen Kongo, ,eines grossen Nimrod“"4; oder an den ersten Menschen Kintu in Uganda, der zugleich der erste Herrscher war und gleich seinem Volke von der Jagd lebte. Weitere Beispiele für Jäger als Herrscherahnen hat Straube ${ }^{5}$ zusammengestellt.

Straube hat übrigens ,ziemlich überzeugend nachgewiesen" ${ }^{\text {"6 }}$ (Ad. E. Jensen, Br. an Verf. vom 17.7.1954), dass das Wesentliche in all diesen Mythen und Traditionen nicht das ist, dass der neue Herrscher ein Land-

${ }^{1} \mathrm{~S}$. Wolf, ,Zum Problem der Frauendarstellungen in der Benin-Kunst“, $A b$ handlungen und Berichte des staatlichen Museums für Völkerkunde, Dresden, 3 r, I970, S. 199 .

${ }^{2} \mathrm{H}$. Baumann, Schöpfung und Urzeit des Menschen im Mythus der afrikanischen Völker, Berlin 1936, S. 251; H. Baumann, ,Zur Frage der Steinbauten und Steingräber in Angola“, Paideuma, 6, 1956, S. 122; D. Westermann, Geschichte Afrikas, Köln I952, S. 352 .

s Schinkel, S. 4I.

4 Baumann, Schöpfung, S. 25 I.

5 H. Straube, Die Tierverkleidungen der afrikanischen Naturvölker (Studien zur Kulturkunde, 13), Wiesbaden I955, S. 57.

- Ad. E. Jensen, Br. an Verf. vom I7.7.1954. 
fremder war, sondern dass es näher liege, ,,den König als den eigentlichen Jäger anzusehen“ (S. 55 f.). Eine allgemeine Entscheidung der Frage in geschichtlichem oder religiös-mythologischem Sinne dürfte kaum möglich sein, besonders da die Vorstellung vom Kultur-Heros auch hereinspielt. Jedenfalls aber liegt die Betonung des fundamentalen jägerischen Zuges des Herrschers vor allem begründet, in seiner Eigenschaft als lebendes Urwesen“1. Dieses sog. „Urwesen“ aber ist, wie wir sehen werden, eine LuzveGestalt. Die existentielle Beziehung zu ihm ist eine dynamische, innere Notwendigkeit.

Nun fällt es auf, wie häufig die Alteingesessenen als Leute geschildert werden, die das Feuer nocht nicht kannten, das ihnen erst die zugewanderten Jäger brachten, worauf jene den Anführer der kleinen Jägerhorde als ihren Häuptling anerkannten². Nach Frobenius hatte das Dziva-Volk im Osten Rhodesiens das Feuerzeug ,verloren“ und nun brachte ihnen der Jäger neues Feuer und wurde ihr Herrscher's. Diese Geschichte gewährt einen Einblick in das, was für eine Bewandtnis es historisch mit dem „Feuerbringer" hat. Es verhält sich nicht so, wie die Mythe oder Tradition es darstellen, als hätte nämlich der alteingesessene Stamm das Feuer überhaupt nicht gekannt, sondern so, dass der Jäger zu ihm als Herrscher mit sein em Feuer kommt - nachdem alle anderen Feuer im Gebiet haben ausgelöscht werden müssen. Das neue Feuer ist das Staatsfeuer des neuen Herrschers. Durch seine Annahme dokumentiert man die Anerkennung des Herrschers oder, anders ausgedrückt, man unterwirft sich ihm, man erkennt seine Zugehörigkeit zum neuen „Staate“ an. Wer die Annahme verweigert, ist ein Rebell.

Von hieraus erklärt sich dann auch die Sitte, dass beim Tode eines Herrschers alle Feuer im Lande ausgelöscht werden müssen und von der Residenz des Herrschers vom heiligen Herde neuentzündetes Feuer nach allen Seiten des Reiches bis in den kleinsten Hof verbreitet wird". Ich sage ,,wird“,

1 Straube, S. 57 .

2 Vgl. v. Sicard, ,Reiche“, S. 657.

${ }^{3}$ L. Frobenius, Erythräa, Berlin u. Zürich I931, S. I I4 f.

4 Frobenius, S. II7, 219; vgl. Haberland, S. 43; Hauenstein, S. 24I ff.; H. v. Sicard, „Herd und Herdfeuer im südlichen Afrika“, Ethnologia Europaea, 4, 197 I, S. II 8 und betrachte dann von hieraus Wolfs "Frauendarstellungen in der BeninKunst"! 
bin mir indessen vollkommen bewusst, dass das ein „Präsens ethnographicum“ ist. Bei den Luba am Luapula bedeutet übrigens -teka I) auf das Feuer setzen, 2) herrschen ${ }^{1}$.

3. Unsere nächste Aufgabe ist es, den Geschehenszusammenhang der mythischen Züge von Feuer und Staat zu ergründen, um dadurch vielleicht dem Verständnis der Geschichte der Urzeit nicht einfach als menschlicher Existenz, sondern als bewusstem, zielstrebigem Handeln näher zu rücken². Was hat Feuer mit dem afrikanischen Staate zu tun? Wie hängen die beiden zusammen? Kann es zwischen ihnen überhaupt einen sinnvollen Zusammenhang geben? Der Europäer mag vor allem den Eindruck gewinnen, dass dieses nach allen Seiten von einem Mittelpunkt aus verbreitete Feuer etwas von menschlicher Zusammengehörigkeit zum Ausdruck bringen will, also im Einklang mit der eingangs erwähnten res publica steht. Ich möchte nicht in Abrede stellen, dass auch diese Seite hier mitklingt. Wir würden uns jedoch in den heute so beliebten sozialen und soziologischen Gesichtspunkten verirren, falls wir den Zug der durch das Feuer zum Ausdruck gebrachten Zusammengehörigkeit zu stark und vor allem auf Kosten anderer Züge betonten.

Das heilige Staatsfeuer wird selbstverständlich nicht mit Streichhölzern entzündet, sondern mit jenem uralten Feuerzeug, das aus zwei Teilen besteht: dem senkrechten zugespitzten Stabe und einem liegenden Holz mit einer Vertiefung in der Mitte. Die Entzündung des Feuers wird als Geschlechtsakt aufgefasst - und wieder liegt die Gefahr eines europäischen Missverständnisses auf der Hand: „Geschlechtsakt? Also sexuelle Handlung! Natürlich! In Afrika wird ja alles zur Sexualität!“ Wer aber hat in diesem Zusammenhang im Mythos vom Staat das Wort "Geschlechtsakt" erfunden? Frobenius sprach vom „natürlichen Sinn seines sexuellen Vorganges" und der „sexuellen Behandlung des Feuerzeuges" (S. 194, II7), betonte jedoch selbst ausdrücklich im Gegensatz hierzu, dass die Königstöchter ,zurückhaltend und keusch" seien und dass der Gesang, der davon spricht, dass die sich hingeben, ,durchaus sakral" sei (S. 193). Damit entkleidet er ihn selbst der Liederlichkeit und der Lust und kommt dadurch der afrikanischen Tatsache unvergleichlich näher. Der Stab heisst auf ka-

\footnotetext{
${ }^{1}$ I. Cunnison, The Luapula Peoples, Manchester I959, S. 217.

2 Vgl. W. Angeli, „Urzeit und Geschichte", Anthropos, 66, I97I, S. 239.
} 
ranga $m u$-siki; Feuer entzünden: -sika moto, und -sika ist dasselbe Verbum, das man für schaffen, erschaffen gebraucht. Gott, Mwari, z. B. ist mu-siki, Schöpfer. Von Sexualität ist hier keine Spur, wohl aber ist hier im höchsten Grade Leben schaffendes Leben versinnbildlicht, das immer stärker ist als der Tod. Europäisiert kommt afrikanisches Staatsfeuer in den Worten zum Ausdruck: „Le roi est mort. Vive le roi!“

4. In afrikanischer Sicht wird der Mythos vom Staat zum Mythos vom Königtum. Ich vermeide es absichtlich, vom ,sakralen Königtum“ zu sprechen, weil dieser Ausdruck zu viele unafrikanische Assoziationen hervorruft. Wohl konstituierte der Herrscher den alten afrikanischen Staat, er konnte jedoch nie Alleinherrscher werden. Nicht einmal der berühmte Shaka war es. In Afrika konnte es nie zum Absolutismus kommen. Dem lebenden afrikanischen Herrscher wurden auch meines Wissens niemals Opfer dargebracht. Auch der Gebrauch des Ausdruckes „Feudalismus“ führt in afrikanischem Zusammenhang leicht zu Missverständnissen, weil das Verhältnis zum Lande dort ein anderes war als bei dem europäischen Feudaladel. Andererseits war die Gewalt des Herrschers durch seine Räte beschränkt. Als Herrscher war er von ihnen und seinen Häuptlingen abhängig. Unbegrenzt dagegen war die Gewalt, die er jenem mythischen -sika zu verdanken hatte. In ihm gipfelte das, was man gerne als „sakrales Königtum" bezeichnet. Der Herrscher war göttlichen Geblüts. Das kommt u. a. deutlich in einigen Ursprungsmythen afrikanischer Herrscherhäuser zum Ausdruck.

Wir berühren hiermit komplizierte religionsgeschichtliche Probleme, die möglicherweise auch auf manche Fragestellung der asiatisch-europäischen Religionsgeschichte umgestaltend einwirken können. Es ist unmöglich, sie mit ein paar Worten abzutun. Vier Beispiele mögen wenigstens andeuten, worum es sich handelt.

I) Der oben erwähnte Kintu, der sagenhafte erste Cwezi im Zwischenseengebiet, heiratete die Himmelstochter Nambi. Von ihnen stammt das Herrschergeschlecht ab. Wichtig für uns ist, dass Kintu manchen Zug mit En-kidu, der babylonischen Ausgestaltung des Herrn der Tiere, teilt. Der Zusammenhang mit dem Zweistromlande wird dadurch erhärtet, dass Kintu-Nambi dasselbe Paar ist wie Setu und seine „Frau“ Nabo bei den Bwaka in nord-westlichen Kongo. Setu und Nabo gehörten zu den ersten 
vier Lebewesen. Leyder identifizierte mit vollem Recht Nabo mit der assyrischen Gottheit gleichen Namens' ${ }^{1}$. In der Bibel begegnen wir den beiden als Adams Sohn Seth, der den Namen Jahwes noch nicht anrief (I. Mos. 4: 25 f.), und Nabo, resp. Nebo (Jes. 46: I).

2) Eine stark christlich gefärbte Legende der Cokwe in Angola weiss zu berichten, dass ihr Herrscherhaus von einem verkrüppelten Sklaven abstamme, dessen sich der Gott Nzambi erbarmte und ihm eine seiner Töchter zum Weibe auf die Erde sandte. Dank der Ehe mit ihr wurde der Lahme geheilt, und die Himmelstochter und der "Sklave“ wurden die Stammeltern der Cokwe-Herrscher ${ }^{2}$.

Wir halten einen Augenblick inne. Es liegt auf der Hand, dass die Mythen vom Ursprung der Herrscherhäuser der Ganda und Cokwe in einem ihrer wesentlichsten Züge derselben Quelle entstammen, obgleich später unterschiedlicher Stoff mit ihnen verbunden worden ist. In dem einen Falle ist die himmlische Urmutter Nambi, im andern ist Nzambi der Schwiegervater. Es ist dies eine mythische Gestalt, die in Afrika nicht mehr einheitlich zu erfassen ist. Als Gottheit scheint sie nicht dasselbe hohe Alter zu besitzen wie einige andere afrikanische Götter ${ }^{3}$, die sie - allem Anschein nach der libysch-kuschitisch-semitischen Sprachenfamilie angehörig - z. T. verdrängt hat. Der bedeutendste Unterschied zwischen beiden Berichten liegt natürlich im männlichen Partner. Dass der Uganda-Bericht Kintu zum Stammvater der Herrscher gemacht hat, nimmt bei seiner überragenden Gestallt nicht Wunder. Die verworrenen und teilweise einander widersprechenden Mythen, die von ihm handeln, legen jedoch den Schluss nahe, dass hinter ihm eine viel ältere mythische Gestalt steht, die von $N z a m b i$ verdrängt wurde und auf die auch Enkidu zurückzuführen ist. Enkidu und der „lahme Sklave“ der Cokwe haben Züge bewahrt, die es uns gerade noch ermöglichen, diese ältere Gestalt zu umreissen.

Es überrascht, dass ein Herrscherhaus nach eigener Tradition von einem Sklaven, der dazu noch ein Krüppel war, abstammt und dass ausgerechnet

${ }^{1} \mathrm{~J}$. Leyder, "Conception des Bwaka sur les astres", Revue de l'Université de Bruxelles, 37, 1934, S. I 5 I, I 54, I $_{5} 8$.

2 V. Martins e H. de Sicard, Contos dos Cokwe, Lisboa I971, No 24.

3 Vgl. H. v. Sicard, „Luwe und verwandte mythische Gestalten“, Anthropos, $63 / 64,1968 / 69$, S. 704 . 
solch ein Sklave die schöne Tochter des Himmelsgottes Nzambi heiratet. Hier stimmt ganz einfach etwas auch für afrikanisches Empfinden nicht. Die Frage drängt sich einem auf: „Wer war eigentlich dieser mythische Herrscherahn?" Ein Vergleich mit entsprechendem afrikanischem religionsgeschichtlichem Material, das ursprünglich nichts mit Königtum zu tun hat, erweist, dass das Schwiegersohnverhältnis des chthonischen Lahmen zum Himmelsgott $N z a m b i$, das durch die Bezeichnung "Sklave" geradezu überbetont wird, wiederholt als Zug einer uralten verdrängten Gottheit aus der Zeit der Sammler, Fallensteller und alten Jäger vorkommt, die wir der Kürze halber zusammenfassend Luwe nennen wollen. Es liegt also Veranlassung vor, im gelähmten Sklaven eine ursprüngliche Luwe-Gestalt der Religion der Jäger der älteren Periode der Saharanischen Kultur ${ }^{1}$ zu vermuten. Luwe war nicht nur Herr der Tiere, der selbst Antilope sein konnte, sondern auch ein atmosphärischer Gott - in einem Märchen ist der Blitz seine Waffe bei einer Auseinandersetzung mit dem himmlischen Schwiegervater ${ }^{2}$.

3) Dieselbe Melodie s. z. s., nur in einer anderen Tonart und mit deutlicherer Akzentuierung, liegt im dritten Beispiel, in einer nicht ganz glücklich als „Swazi"-Märchen bezeichneten Erzählung" vor. Die Rollen sind hier vertauscht. Eine wunderbare Antilope lockt ein verachtetes irdisches Mädchen immer höher in die Berge. Dort verwandelt sie sich in einen Jüngling, den Herrn der Tiere. Das Mädchen hilft ihm, für seine ständig wachsende Antilopenherde zu sorgen, die sich zuletzt in eine grosse Schar von Männern, Frauen und Kindern verwandelt, deren Häuptling der Jüngling ist $t^{4}$. - Es ist nichts anderes als eine südliche Variante zu Nyi-kang, dem mythischen Reichsgründer der Shilluk am Weissen Nil, welcher Herr der Tiere und des Wassers war $^{5}$ und den bis heute besonders die Antilope vertritt ${ }^{6}$.

\footnotetext{
${ }^{1}$ ca. 8000-4000 v. Chr.; vgl. J. Bernolles, ,Les Noirs et le Sahara", Études Da= homéennes, N.S. 4, 1965, S. 69 ff.; Anthropos, 60, 1965, S. 861 f.

2 H. v. Sicard, Ngano dzeciKaranga / Karanga-Märchen (Studia Ethnographica Upsaliensia, 23), Lund 1965, S. 2 I $_{3}$ f.; v. Sicard, ,Luzve", S. 709.

3 E. J. Bourhill and J. B. Drake, Fairy Tales from South Africa, London I908, S. $212 \mathrm{ff}$.

4 Vgl. v. Sicard, „Luzve“, S. 682: 62.

${ }^{5}$ H. Tegnaeus, Le héros civilisateur (Studia Ethnographica Upsaliensia, 2), Uppsala I950, S. $122 \mathrm{f}$.

6 Straube, S. 45.
} 
4) Unsere letzte Variante liegt geographisch zwar ausserhalb des eigentlichen Bantu-Gebietes, liefert jedoch in kulturgeschichtlicher Hinsicht zur Frage "Mythe und Staat" so grundlegende Erkenntnisse, dass sie hier einbezogen werden muss. Sie stammt aus Benin mit seinem sog. ,sakralen Königtum“, dass die europäische „Scheidung des Kultischen vom Profanen erschwert oder unmöglich macht" ${ }^{\prime 1}$ und handelt vom Oba (Herrscher) Ohen, der gegen ${ }^{3} 33^{\circ}$ regiert haben soll2.

Interessant ist nun in Benin mit seinen zahllosen Klangottheiten nicht nur die Verschmelzung des Oba im allgemeinen, sondern ganz besonders diejenige des Oba Ohen mit der Gottheit Olókun, welche von ,vorrangiger Bedeutung " ist ${ }^{3}$. Ehe wir jedoch näher auf dieses Problem eingehen, müssen wir uns mit der Frage auseinanderzusetzen suchen, wer Olókun eigentlich war und wie die Benin-Leute und die Yoruba und Ife, mit denen sie verwandt sind, zu ihm kamen? Ganz abgesehen vom Fehlen oder von der Unzuverlässigkeit älterer Quellen - arabische Geographen und Händler des Mittelalters - gehören historische Einzelheiten nicht in diesen Zusammenhang. Vermerkt sei nur, dass die Yoruba-Sprache seit mehreren Jahrtausenden ("several thousands of years") in ihrem jetzigen Gebiet gesprochen sein soll', dass die Yoruba-Völker selbst aus dem Nordosten gekommen sein wollen ${ }^{5}$, dass die einheimischen Traditionen auf eine Wanderung ihrer Königskultur vom Čad-See her verweisen ${ }^{6}$ und dass H. Baumann schon 1936 (S. I34 f.) das Empfinden hatte, dass die Mythik der Yoruba sich „derart stark" von der ,allgemein-afrikanischen“ unterscheide, dass sie den Blick unwillkürlich auf das Mittelmeer lenke. Voller Überzeugung schrieb er schon damals: „Die Wurzeln dieser Kultur liegen weit im Norden - wohl im alten vorindogermanischen Mittelmeergebiet" (S. 132). Olderogge und Potechin ${ }^{7}$ wiederum geben u. a. die Meinung einiger Forscher wieder, der zufolge die Guinea-Kultur mit Napata und Meroë zu

1 Wolf, S. 200.

2 Wolf, S. 209: 37; Westermann, S. 269.

3 Wolf, S. 215 .

4 W. Fagg, Nigerian Images, London 1963 , S. 25, 30.

5 Westermann, S. 252.

- K. Dittmar, ,Zur Herkunft und Bedeutung der altyorubischen Kronen und der äthiopischen Kalatscha", Festschrift für Ad. E. Fensen, x, 1964, S. 85.

- Narody Afriki, red. D. A. Olderogge i I. I. Potechin, Moskva I954, S. 276. 
verbinden sei. Selbst weisen sie auf die Terrakotta-Funde von Nok hin, die jetzt auf die Zeit zwischen ca. $500 \mathrm{v}$. Chr. und $200 \mathrm{n}$. Chr. datiert werden 1 . Nok liegt im Norden Nigerias, einige Hundert Kilometer westlich vom $\breve{C a d-S e e, ~ w o ~ F u n d e ~ e i n e r ~ f r u ̈ h e n ~ K u l t u r, ~ u . ~ a . ~ L e h m s t a t u e t t e n, ~ a n ~ E r z e u g-~}$ nisse der altmediterranen Kultur erinnern'2.

$\mathrm{Zu}$ den annähernd 400 vergotteten Ahnen der Yoruba-Völker werden auch einige Gottheiten gerechnet, was das von Verfall zeugende Gebilde ihrer Mythologie ${ }^{3}$ noch mehr verwickelt und eindeutig von einem Konglomerat zeugt. Als Belege seien nur die Götter Olókun und Shango erwähnt. Die Entwirrung dieses Pantheons ist übrigens eine sehr dringende Aufgabe der Forschung, da sie zur Ermöglichung einer Unterscheidung von Kulturschichten einen wesentlichen Beitrag liefern würde.

Bezüglich Shango sei hier nur hervorgehoben, dass er ein Doppelgänger Olókuns ist ${ }^{4}$. Das erklärt nicht nur so gut wie alle seine Eigenschaften und Attribute, sondern u. a. auch die Mythe, nach der er einer der ersten Herrscher der Yoruba gewesen sein soll. Die Oyo nämlich, die in der ersten Hälfte des I2. Jh:s aus dem Norden ins Land kamen, führen ihren Ursprung auf „König Shango“ zurück, der „später mit dem Gewittergott gleichgesetzt" wurde und stets als Reiter dargestellt wird". Dass er auf dieselbe mythische Gestalt zurückgeht wie der oben erwähnte nilotische $N y i$ kang, kann mit Bestimmtheit angenommen werden. Man denke jedoch auch an das ,Swazi"-Märchen und an Luwwes Reittier, die Antilope.

Damit aber ist auch schon das Wesentlichste über Olókun ausgesagt. Das Verhältnis dieser Doppelgestalt zum ,urelterlichen Götterpaar“ ist in der heutigen Mythologie äusserst widerspruchsvoll. Einmal ist es Shango, der seinen „Vater" Olórun verdrängt hat, ein anderes Mal wird er mit Olórun identifiziert; dann wieder ist der „Meergott" Olókun mit Olórun ,gleichaltrig"6. Baumann hat $O-l o ́ k-u n$ in sein Verzeichnis westafrikanischer Göt-

\footnotetext{
${ }^{1}$ Fagg, S. 23; vgl. D. A. Olderogge, Iskusstvo zapadnoj Afriki v muzejah SSSR, Leningrad-Moskva I958, S. 7.

2 Olderogge, Iskusstvo, S. 8.

3 Fagg, S. $27 \mathrm{f}$.

${ }^{4}$ Vgl. v. Sicard, „Luzve", S. 677, 70I: ,Mu-tanga /= Shango/ ein anderes Wort für $M u$-lungu'" $/=O$-lókun/.

${ }^{5}$ Dittmar, S. 88.

- Baumann, Schöpfung, S. I34.
} 
ternamen mit dem Stamm -lung-, -luk-: ,Meer"1 nicht aufgenommen, da es keine etymologische Studie zu sein beansprucht. Tut man es aber, dann gewinnt man neue und überraschende Einblicke in alte religionsgeschichtliche Zusammenhänge und in den afrikanischen Mythos vom Staat.

Das reichhaltigste Material über Olókun und das Königtum verdanken wir jetzt Siegfr. Wolf². Zu beachten ist, dass Olókun in der Mythologie der Bini heute nicht die höchste Stellung einnimmt. Diese kommt vielmehr dem „Schöpfergott Osa“ zu. Olókun ist dessen ,ältester Sohn“ und zugleich einer seiner wichtigsten Ratgeber. Olókun-Aspekte und -Symbole wurden in der Benin-Plastik ,,immer wieder zu bevorzugten, von den Konsumenten geforderten" Themen3. Er ist die in Benin populärste Gottheit (S. 200), hat aber diese "herausgehobene Stellung im religiösen Leben der Bini“ nicht erst „erlangt“ (von mir gesperrt), wie Wolf das ausdrückt (S. 213), sondern ist die z. T. verdrängte und den Gottheiten späterer Einwanderer untergeordnete älteste Gottheit, deren Namen und Wesen der afro-eurasiatische Kontinent seit der Zeit der Sammler und Fallensteller und später auch der Jäger in verschiedenen Varianten durch Abstossung und Assimilierung bis auf unsere Zeit bewahrt hat. Vergessen wir nicht, dass das Yoruba-Königtum über die Bini eine Fremdherrschaft ist ${ }^{\underline{4}}$. Olókun als älteste Gottheit in dem Gebiet der Yoruba-Staaten hat sich immer wieder so siegreich zu behaupten gewusst, dass er sogar ,als Vertreter des höchsten Gottes"5 fungieren kann! Und gerade mit dieser Gottheit behaupten nun verschiedene afrikanische Herrschergeschlechter zusammenzuhängen, u. a. gewissermassen auch dasjenige von Benin.

Wenn Olókun dazu noch geschlechtlich ambivalent ist ${ }^{6}$, so hebt das noch stärker seine Identität mit dem nordischen Loki und mit Afrikas Luwe hervor? Dazu kommt noch der folgende entscheidende Zug, den Wolf nur wie von ungefähr erwähnt: „Nicht nur Tiere des Wassers sind ihm untertan, sondern auch landbewohnende können ihm zugehören." Olókun ist

1 Baumann, Schöpfung, S. 89.

2 Wolf, ,Zum Problem der Frauendarstellungen in der Benin-Kunst".

3 Wolf, S. 207.

4 Fagg, S. 3 I.

${ }_{5}$ Wolf, S. 213 .

6 Wolf, S. $209,{ }^{7} 2 \mathrm{I} 6$.

"v. Sicard, ,Luwe“, S. 689, 706. 
also auch Herr der Tiere, und in einer Anmerkung (S. 213/48) wird als sein Tier ausdrücklich das ,king deer“ erwähnt, eine Antilopengattung, dit mit "blackfronted duiker" widergegeben wird. Es ist Luwes Reittier kat' exochèn, nur dass es in Olókuns Fall in Benin als Verfallserscheinung "teils in der See lebend gedacht ist".

Olókuns Zusammenhang mit dem Königtum enthält dann noch in einen weiteren wichtigen Luwe-Zug in Erscheinung. Die Luzve-Gestalt tritt man sich oft als halbseitig oder hinkend vor. Der Urahn der Cokwe-Herrscher war, wie wir gesehen haben, ,gelähmt", und nun heisst es vom oben erwähnten $\mathrm{Oba}$ Ohen, dass er ,eine Beinlähmung erlitt", andererseits aber auch, dass er „vom Gott Olókun besessen gewesen sei" und seinen Kult eingeführt habe oder, wie eine andere Quelle es ausdrückt, dass er ,gave out that he had become the sea god"1. Bronzene Reliefs, die Oba Ohen darstellen sollen, zeigen ihn als welsbeinige Gestalt, die von zwei Häuptlingen gestützt wird². Man beachte das Flechtbandornament auf der Beinbekleidung des einen Häuptlings. Es ist in der Beniner Kunst äusserst beliebt und tritt, seinem Symbolwert gemäss, besonders auf Darstellungen auf, die es mit der Lebensförderung zu tun haben. Es ist ein Zug der altmediterranen Kultur, der jedoch auch in den Kreis der Luwe-Vorstellungen einbezogen worden ist ${ }^{3}$.

\section{III.}

Es nimmt nicht Wunder, dass das afrikanische Königtum gerne an eine der ältesten, chthonisch bestimmten Gottheiten aus der Zeit vor dem Austrocknen der Sahara (4000-3000 v. Chr.) anknüpft, bevor die grossen Wanderungen nach allen Richtungen einsetzten, ,la fuite du désert", wie es A. C. Blanc ${ }^{4}$ so treffend ausgedrückt hat, u. a. an die Guinea-Küste und in die Gebiete des östlichen Mittelmeeres.

\footnotetext{
1 W. Fagg and M. Plass, African sculpture, an anthology, London \& New York I964, zitiert bei Wolf, S. 215:59.

${ }_{2}$ Fagg, Taf. 16; Wolf, S. 215.

3 Vgl. v. Sicard, ,Luwve“, S. 691: 6 mit weiteren Hinweisen; H. Baumann, „Die ethnologische Beurteilung einer vorgeschichtlichen Keramik in Mittelafrika", Festschrift fïr Ad. E. Yensen, I, I964, S. 29 ff.; Dittmar, S. 68, 78, 85.

"A. C. Blanc, ,Sur le facteur fondamental des mouvements des cultures pré- et protohistoriques en Afrique du Nord: La fuite du désert ", Prehistoric Art of the Western Mediterranean and the Sahara, ed. by L. Pericot Garcia and E. Ripoll Perello (Viking Found Publications in Anthropology, 39), 1964, S. 179, I81.
} 
Bei der Behandlung dieses urtümlichsten Mythos vom Staat kann man noch gar nicht von Makrokosmos und Mikrokosmos und von astral-mythologisch begründetem sakralem Herrschertum reden, etwa von Sonnenkönigen oder von Mondgottkönigen mit ihren Venusfrauen vom Typus Monomotapa, wie das für jüngere altorientalische und altmediterrane Kulturen und ihre Ausläufer - auch in Afrika - angemessen ist. Die Kettenfolge geschichtlicher Tatsachen darf hier nicht verwischt, kulturstratigraphische Feststellungen nicht durcheinandergeworfen werden, indem man unbedachtsam bei der altmediterranen Kultur und ihren Einflüssen stehen bleibt.

Wir haben oben die ältere Periode der Saharanischen Kultur in Erwägung gezogen (ca. 800o-400o v. Chr.). Während dieser Periode dürften die zahlreichsten Bewohner der bis zu einem gewissen Grade blühenden Sahara Pygmäen und schwarze paläonegritische Sippen und Sippschaften gewesen sein, die zu Beginn des folgenden Jahrtausends, als das gewaltige Gebiet sich in eine Wüste zu verwandeln begann, zum grössten Teil in südlicher Richtung auswanderte. Dieser Rückzug geschah jedoch auch unter dem Druck einer neuen Bevölkerungswelle, mit der nach unseren heutigen Kenntnissen die zweite grosse Periode der Saharanischen Kultur beginnt. Im Gegensatz zu den ursprünglichen Bewohnern des Gebietes, die Sammler, Fallensteller und Jäger waren, sind die neuen Ankömlinge Viehzüchter, was natürlich Ackerbau in beschränktem Masse in beiden Schichten nicht unwillkürlich auszuschliessen braucht. Die neu zugewanderten Hirten waren hellhäutig. Bernolles ( 1965$)$ vermutet, dass sie aus Südarabien kamen und anfangs, sei es zwischen Åthiopien und Ägypten, sei es im Gebiet von Uganda, ein Nomadenleben führten ${ }^{1}$. R. Herzog ${ }^{2}$ verlegt Punt, das ,schon“ im 15. Jh. v. Chr. von Negroiden, die „durch Hellfarbige (wohl Kuschiten) überschichtet" waren und das in der Nähe des "Schweifgebietes" von Pygmäen lag, in die Republik Sudan bzw. in angrenzende Landschaften am Weissen oder Blauen Nil oder am Atbara ${ }^{3}$.

\footnotetext{
1 Vgl. Anthropos, 6o, 1965, S. 862.

2 Vgl. Anthropos, 66, I 97 I, S. 246.

${ }^{3}$ Eine andere Hypothese bei R. et M. Cornevin, Histoire de l'Afrique (Petite bibliothèque Payot, 57), Paris 1964, S. 71; unsere Schlussfolgerung verändert sie nicht.
} 
Schon diese Viehzüchter brachten nach Afrika, wenn auch unbewusst, religiöses Lebensgut und alte Kulturgüter in teilweise umgestalteter Form zurück, die einmal von den ältesten Trägern der Saharanischen Kultur aus in die beiden anderen Teile des Trippelkontinentes gelangt waren, und wir werden kaum fehlgehen, wenn wir zu diesen vor allem den Glauben an den chthonischen Herrn der Tiere, des Wassers, des Regens und des blitzenden Feuers und damit des Jenseits; ferner die ersten Anfänge eines göttlich bestimmten Führertums, und die mythische Bedeutung der Hörner, in denen Lebenskraft und Führertum ihren symbolischen Ausdruck fanden, rechnen. Führertum kam sogar bei den Jägern, die die ursprünglichen Krieger sind $^{1}$ stärker zum Ausdruck als bei den Viehzüchtern, die wir im Hinblick auf das gezähmte Wild vielleicht als ,nichtjagende Jäger" bezeichnen dürfen.

Die Verbindung mit der Aussenwelt riss natürlich auch während des Jahrtausends des Austrocknens der Sahara nicht ab, und in den folgenden Jahrtausenden machten sich die Einflüsse der sog. altmediterranen Kultur einerseits in Nubien ${ }^{2}$, andererseits dank der ,route des chars du Sahara central" von Libyen ${ }^{3}$ durch das Gebiet der Tuareg-Berber mit dem Sudan der Schwarzen bemerkbar und trugen in einer neuen Zeit dazu bei, auf der Grundlage der alten Luwe-Herrschermythen ein modernes, altmediterran bestimmtes afrikanisches Königtum mit seiner Mythologie auszugestalten, das bis in unsere Zeit unter den Bantu-Völkern weiterlebt.

${ }^{1}$ Vgl. z. B. Dittmar, S. 73; S. Donadoni, ,Remarks about Egyptian Connections of the Sahara Rock Shelter Art", Prehistoric Art of the Mediterranean and the Sahara, ed. by L. Pericot Garcia and E. Ripoll Perello (Viking Found Publications in Anthro= pology, 39), r964, S. I86.

${ }^{2}$ Dittmar, S. 80, 84: 23 rechnet mit der Möglichkeit einer ,-, noch unbekannten - nubischen Kultur"!

a Cornevin, S. 70 f.; Dittmar, S. 8r: rg. 